\title{
INTERVALO HÍDRICO ÓTIMO EM UM LATOSSOLO VERMELHO CULTIVADO EM SISTEMA SEMEADURA DIRETA POR 25 ANOS $^{(1)}$
}

\author{
Fernando Rodrigues Moreira ${ }^{(2)}$, Sonia Carmela Falci Dechen ${ }^{(3)}$, Álvaro Pires da Silva ${ }^{(4)}$, \\ Getulio Coutinho Figueiredo ${ }^{(5)}$, Isabella Clerici De Maria ${ }^{(3)}$ \& Patrícia Terezinha \\ Pessoni ${ }^{(2)}$
}

\begin{abstract}
RESUMO
O Intervalo Hídrico Ótimo (IHO) integra atributos físicos do solo relacionados ao crescimento das culturas e corresponde ao intervalo entre os limites superior e inferior do conteúdo de água no solo, no qual são mínimas as limitações para o crescimento radicular. Em áreas agrícolas, o manejo do solo pode alterar sua estrutura, de forma que, principalmente em razão da compactação, a densidade do solo (Ds) poderá ficar fora desses limites em que as condições são ideais para o crescimento das plantas. O objetivo deste trabalho foi utilizar o IHO para avaliação da qualidade física do solo e identificação de áreas com restrição para crescimento de plantas, visando ao manejo localizado. $O$ estudo foi realizado em um Latossolo Vermelho argiloso sob sistema semeadura direta (SSD) com cultivos sucessivos de grãos por 25 anos em Campinas, SP. Foram coletadas amostras volumétricas de solo, nas camadas de $0,00-0,10,0,10-0,20$ e $0,20-0,30 \mathrm{~m}$, destinadas à obtenção dos seguintes atributos: Ds, curva de resistência à penetração, curva de retenção de água e porosidade do solo para determinação do IHO e da densidade crítica do solo (Dsc). Dados de variabilidade e dependência espacial da Ds foram analisados por semivariogramas para elaboração de mapas desse atributo. O IHO diminuiu em profundidade e foi limitado na parte superior pela umidade na capacidade de campo e na parte inferior pela resistência à penetração, nas três profundidades avaliadas.
\end{abstract}

(1) Parte da Dissertação de Mestrado do primeiro autor. Recebido para publicação em 20 de junho de 2012 e aprovado em 5 de setembro de 2013.

(2) Engenheiro Agrônomo, Mestre pelo Programa Pós-Graduação em Agricultura Tropical e Subtropical do Instituto Agronômico - IAC. Av. Barão de Itapura, 1481. CEP 13020-902 Campinas (SP). Bolsista FAPESP. E-mail: fernandolpp@yahoo.com.br, patriciapessoni@hotmail.com

(3) Pesquisador Científico, Centro de P\&D de Solos e Recursos Ambientais, IAC/APTA/SAA. Av. Barão de Itapura, 1481. CEP 13020-902 Campinas, (SP). E-mail: dechen@iac.sp.gov.br, icdmaria@iac.sp.gov.br

(4) Professor do Departamento de Ciência do Solo, Escola Superior de Agricultura "Luiz de Queiroz", Universidade de São Paulo - ESALQ/ USP. Av. Pádua Dias, 11. CEP 13418-900 Piracicaba (SP). E-mail: apisilva@usp.br

(5) Engenheiro Agrônomo, Doutor em Ciências pelo Programa Pós-Graduação em Solos e Nutrição de Plantas da ESALQ/USP. Av. Barão de Itapura, 1481. CEP 13020-902 Campinas (SP). Bolsista FUNDAG. E-mail: figueiredo.gc@gmail.com 


\begin{abstract}
A Dsc foi de 1,42 $\mathrm{Mg} \mathrm{m}^{-3}$ para a camada de 0,00-0,10 m e de 1,39 e 1,41 $\mathrm{Mg} \mathrm{m}^{-3}$, respectivamente, para as camadas de 0,10-0,20 e 0,20-0,30 m. Verificou-se que a Ds foi maior que a Dsc nas camadas de 0,00-0,10 e 0,10-0,20 m, em porções localizadas no terço inferior do terreno, indicando condição crítica para o crescimento das plantas. A utilização do IHO, associado a mapas de variabilidade espacial da Ds, para determinação de pontos em que ela é maior que a Dsc, auxilia a tomada de decisão para intervenção ou modificação do manejo do solo, enquanto que o critério de escolha do valor crítico da resistência à penetração pode contribuir para a interpretação dos resultados de campo.
\end{abstract}

Termos de indexação: água no solo, plantio direto, qualidade física do solo.

\title{
SUMMARY: LEAST LIMITING WATER RANGE IN AN OXISOL CULTIVATED UNDER A NO-TILLAGE SYSTEM FOR 25 YEARS
}

\begin{abstract}
Least Limiting Water Range (LLWR) integrates soil physical properties related to crop growth and corresponds to the interval between the upper and lower limits of water content in the soil within which limitations to root growth are minimal. In agricultural areas, soil management can lead to changes in its structure, mainly due to compaction and, subsequently, bulk density can reach values outside the limits in which conditions are ideal for plant growth. Therefore, the aim of this study was to use the LLWR to evaluate soil physical quality and to identify areas that restrict plant growth, with a view toward localized management. This study was carried out using the LLWR to evaluate soil physical quality so as to identify areas in which bulk density is higher than critical bulk density in an Oxisol under no-tillage system since 1985, in Campinas, São Paulo, Brazil. Undisturbed samples were collected at the depths of 0.00-0.10, 0.10-0.20 and 0.20-0.30 m to obtain the following attributes: bulk density, penetration resistance curve, water retention curve and soil porosity. Data on spatial variability of bulk density and critical bulk density were analyzed by semivariograms to map these attributes. The LLWR decreased in depth and was limited at the top by the moisture at field capacity and at the bottom by resistance to penetration at the three depths studied. Critical soil density was $1.42 \mathrm{Mg} \mathrm{m}^{-3}$ for the 0.00-0.10 m layer and 1.39 and $1.41 \mathrm{Mg} \mathrm{m}^{-3}$, respectively, for the 0.10-0.20 and 0.20-0.30 m layers. It was observed that soil bulk density was higher than critical soil density in the 0.00-0.10 and 0.10-0.20 m layers in the lower portions of the field, indicating a critical condition for plant growth. The use of LLWR, associated to maps of spatial variability of soil bulk density to determine points at which it is higher than critical soil bulk density aids decision making for intervention or modification of soil tillage, while the selection criterion of the critical value of penetration resistance can contribute to the interpretation of field results.
\end{abstract}

Index terms: soil moisture, no-tillage, soil physical quality.

\section{INTRODUÇÃO}

A gestão de recursos agroambientais é fundamental para o desenvolvimento da agricultura sustentável, e o estabelecimento de métodos para a avaliação da qualidade física do solo torna-se cada vez mais necessário. Esses métodos devem integrar diversas propriedades do solo que expressem o ambiente onde as culturas são desenvolvidas (Doran \& Parkin, 1994).

O Intervalo Hídrico Ótimo (IHO), ou "Least Limiting Water Range" (LLWR), integra propriedades do solo diretamente relacionadas com o desenvolvimento das culturas e corresponde ao intervalo entre os limites superior e inferior do conteúdo de água no solo, no qual são mínimas as limitações para o desenvolvimento radicular (Silva et al., 1994; Beutler et al., 2004; Leão et al., 2004; Klein \& Camara, 2007; Freddi et al., 2008). O limite superior é definido pela capacidade de campo ou pela porosidade de aeração, enquanto o limite inferior, pelo ponto de murcha permanente ou pela umidade em que a resistência do solo à penetração $(\mathrm{RP})$ atinge valores críticos ao desenvolvimento das raízes (Silva et al., 1994; Tormena et al., 1998b). Com o aumento da densidade do solo (Ds) ocorre, geralmente, diminuição do valor do IHO, podendo este atingir o valor zero quando os limites superiores e inferiores são numericamente iguais. Nessas condições, a Ds é denominada como densidade crítica do solo (Dsc) ao desenvolvimento das plantas (Silva et al., 1994; Imhoff et al., 2001).

Diferentes autores têm considerado o valor de 2 MPa (Klein \& Libardi, 2000; Beutler et al., 2004; Leão et al., 2004) como crítico ao crescimento das raízes, porém a utilização desse valor tem sido discutida por outros autores, como Klein \& Camara (2007) e 
Calonego et al. (2011). De acordo com Calonego et al. (2011), em sistemas com semeadura direta (SSD), em razão de os canais deixados com a morte das raízes, os quais orientam o crescimento e desenvolvimento radicular em profundidade, a $R P$ crítica ao crescimento das culturas pode ser superior ao limite considerado em sistemas convencionais de manejo, em que a mobilização do solo causaria a interrupção da continuidade dos macroporos e dos canais do solo. De Maria et al. (1999) observaram maior densidade de raízes em semeadura direta, ainda que esse sistema apresentasse maiores Ds e RP, comparativamente ao preparo convencional do solo.

Sistemas de semeadura direta são sistemas conservacionistas em que a movimentação do solo é a mínima possível e a manutenção dos restos culturais na superfície do terreno contribui para a melhoria de propriedades físicas do solo (Costa et al., 2003; Bertol et al., 2004), favorecendo a infiltração da água e diminuindo a erosão. No entanto, sob SSD, a menor movimentação do solo associada ao tráfego de máquinas proporciona aumento da $\mathrm{Ds}$ e da microporosidade e redução da macroporosidade na camada mais superficial do solo (Tormena et al., 1998a; Cavalieri et al., 2006; Pereira et al., 2010), determinando menores valores do IHO e ocorrências mais frequentes de $\mathrm{Ds} \geq \mathrm{Dsc}$.

Estudos que agregam maior conhecimento a respeito do IHO e da Dsc de áreas em SSD consolidado merecem destaque, visto a necessidade de conhecimento a respeito da qualidade física de solos agrícolas submetidos a sistemas de manejo conservacionista de longa duração. A importância da avaliação da qualidade do solo e identificação de áreas com atributos físicos limitantes no campo está relacionada com a possibilidade de realização de manejo diferenciado, utilizando ferramentas de agricultura de precisão. O objetivo deste trabalho foi avaliar o intervalo hídrico ótimo em um Latossolo Vermelho argiloso, sob SSD por 25 anos, considerando-se 2 e $3 \mathrm{MPa}$ como valores de resistência à penetração, para identificar seu efeito no limite inferior do IHO e na decisão de operações de manejo diferenciado.

\section{MATERIAL E MÉTODOS}

O estudo foi desenvolvido no Centro Experimental Central (CEC) do Instituto Agronômico (IAC), em Campinas, SP, em um campo de 3,6 ha, em um Latossolo Vermelho férrico (Santos et al., 2006), textura argilosa, com declividade média de $10 \%$. As coordenadas geográficas da área são $22^{\circ} 53^{\prime}$ de latitude $\mathrm{S}$ e $47^{\circ} 04^{\prime}$ de longitude $\mathrm{W}$, e o clima, de acordo com Köppen, é do tipo Cwa, com temperatura média entre 18 e $22^{\circ} \mathrm{C}$ e precipitação pluvial anual de $1.385 \mathrm{~mm}$ (Setzer, 1966). A área de estudo permaneceu em pousio entre 1975 e 1985 e, em março de 1985, foi inteiramente limpa, arada e semeada com Crotalaria juncea, sem nenhum adubo ou corretivo. Em seguida, a área foi subsolada, arada, gradeada e teve sua acidez corrigida com a aplicação de $4 \mathrm{t} \mathrm{ha}^{-1}$ de calcário. Em novembro de 1985 a área foi semeada com soja e desde então tem sido manejada sob sistema semeadura direta (SSD) com sucessão de culturas de grãos no verão (soja e milho) e inverno (aveia e triticale), tendo sido cultivados eventualmente arroz, crotalária, lablabe, milheto e mamona. A área recebeu calagem em superfície e fertilizantes, conforme a análise de solo e recomendações técnicas das culturas.

Foram estabelecidos 102 pontos de amostragem em uma grade de $30 \times 30 \mathrm{~m}$. Em fevereiro de 2011, em cada um dos pontos, nas camadas de $0,00-0,10,0,10$ 0,20 e $0,20-0,30 \mathrm{~m}$, foram coletadas amostras indeformadas com o auxílio de cilindros de aço inoxidável de $100 \mathrm{~cm}^{3}(0,05 \mathrm{~m}$ de diâmetro e $0,05 \mathrm{~m}$ de altura). Adicionalmente, coletaram-se mais 18 amostras por camada, totalizando-se, assim, 120 amostras por profundidade.

Para a determinação das curvas da resistência do solo à penetração e da curva de retenção de água no solo, as amostras foram saturadas pela elevação gradual de uma lâmina de água numa bandeja, pelo período de $48 \mathrm{~h}$, até atingir $2 / 3$ da altura do anel. Dividiram-se as amostras em nove grupos com 36 repetições, sendo 12 por profundidade, e cada grupo de amostras foi submetido a uma das seguintes tensões: 2, 4, 6 e $10 \mathrm{kPa}$, em mesas de tensão, e 30, $50,100,500$ e $1.500 \mathrm{kPa}$, em câmaras de Richards. Após o equilíbrio em cada tensão, as amostras foram pesadas, para cálculo da umidade volumétrica. Em seguida, foi determinada a resistência do solo à penetração, em cada amostra, com a utilização de um penetrômetro descrito por Tormena et al. (1998b). O penetrômetro possui uma célula de carga, com capacidade nominal de $20 \mathrm{~kg}$, associada a uma haste com ponta cônica (semiângulo de $30^{\circ} \mathrm{e}$ área basal de $0,1167 \mathrm{~cm}^{2}$ ) e velocidade de penetração de $0,167 \mathrm{~mm} \mathrm{~s}^{-1}$.

A resistência do solo à penetração foi registrada por um sistema automático de aquisição de dados. Em cada amostra, foram descartadas as primeiras e as últimas leituras, ficando apenas as informações dos 3 cm centrais de cada amostra. Em seguida, as amostras foram secas em estufa a $105^{\circ} \mathrm{C}$, por $24 \mathrm{~h}$, para determinação do conteúdo de água e da Ds.

Para determinar o IHO, foi utilizado o método estabelecido por Silva et al. (1994), conforme a descrição que se segue.

A curva de resistência do solo à penetração foi ajustada com base no modelo proposto por Busscher (1990) e descrita pela equação:

$$
R P=a \theta^{b} D^{c}
$$

em que RP é a resistência do solo à penetração $(\mathrm{MPa})$; $\theta$, o conteúdo volumétrico de água $\left(\mathrm{m}^{3} \mathrm{~m}^{-3}\right)$; Ds, a densidade do solo ( $\left.\mathrm{Mg} \mathrm{m}^{-3}\right)$; e a, b, c são os parâmetros de ajuste do modelo. 
Com o ajuste da curva de resistência do solo à penetração, determinou-se o $\theta$ em que a RP atinge os valores críticos de 2 e $3 \mathrm{MPa}$ em função da densidade do solo, rearranjando-se a equação 1:

$$
\theta_{\mathrm{RP}}=\left[\mathrm{RPcrítica} /\left(\mathrm{aDs}^{\mathrm{c}}\right)\right]^{1 / \mathrm{b}}
$$

em que $\theta_{R P}$ ó o conteúdo de água no solo em que a RP atinge o valor crítico; RPcrítica, a resistência crítica do solo à penetração.

A curva de retenção de água foi ajustada por meio do modelo não-linear, descrito na equação:

$$
\theta=\exp (\mathrm{d}+\mathrm{eDs}) \psi^{\mathrm{f}}
$$

em que $\theta$ é expresso em $\mathrm{m}^{3} \mathrm{~m}^{-3} \mathrm{e} \mathrm{Ds}$, em $\mathrm{Mg} \mathrm{m}^{-3} ; \psi$, o potencial mátrico aplicado $(\mathrm{kPa})$; e d, e, f são os parâmetros de ajuste do modelo.

Considerou-se a capacidade de campo $\left(\theta_{\mathrm{CC}}\right)$ como o $\theta$ no potencial mátrico de $-10 \mathrm{kPa}$, e o ponto de murcha permanente $\left(\theta_{\mathrm{PMP}}\right)$ como o $\theta$ no potencial mátrico de $-1.500 \mathrm{kPa}$. Foram calculados os valores críticos do conteúdo de água no $\theta_{\mathrm{CC}}$ e no $\theta_{\mathrm{PMP}}$ pelas seguintes equações:

$$
\begin{gathered}
\theta_{\mathrm{CC}}=\exp (\mathrm{d}+\mathrm{eDs}) 100^{\mathrm{f}} \\
\theta_{\mathrm{PMP}}=\exp (\mathrm{d}+\mathrm{eDs}) 15.000^{\mathrm{f}}
\end{gathered}
$$

em que $\theta_{\mathrm{CC}}$ e $\theta_{\text {PMP }}$ são expressos em $\mathrm{m}^{3} \mathrm{~m}^{-3}$; e d, e, $\mathrm{f}$ são os parâmetros de ajuste do modelo.

O conteúdo de água em que a porosidade de aeração do solo é de $0,1 \mathrm{~m}^{3} \mathrm{~m}^{-3}\left(\theta_{\mathrm{PA}}\right)$ foi descrito pela equação:

$$
\theta_{\mathrm{PA}}=(1-\mathrm{Ds} / \mathrm{Dp})-0,1
$$

em que $\theta_{\mathrm{PA}}$ é expresso em $\mathrm{m}^{3} \mathrm{~m}^{-3} \mathrm{e}$ Dp é a densidade de partículas do solo $\left(\mathrm{Mg} \mathrm{m}^{-3}\right)$. A Dp foi determinada em cada amostra, utilizando-se um picnômetro a gás Hélio.

A densidade crítica do solo (Dsc) ao desenvolvimento das culturas foi determinada quando os limites superior e inferior do IHO foram numericamente iguais.

A variabilidade espacial da Ds e a da Dsc foram analisadas por meio de métodos geoestatísticos, calculando-se a semivariância (Vieira, 2000). Os mapas dos valores das variáveis foram construídos interpolando os dados originais por krigagem, método estatístico em que as estimativas são feitas sem tendência e com variância mínima, utilizando os parâmetros dos semivariogramas. O programa Surfer ${ }^{\circledR}$ versão 9.0 ("Golden Software") foi utilizado para a manipulação e a visualização da distribuição espacial, por meio da construção dos mapas.

\section{RESULTADOS E DISCUSSÃO}

Os valores dos atributos físicos do solo nas camadas de $0,00-0,10,0,10-0,20$ e $0,20-0,30 \mathrm{~m}$ estão apresentados no quadro 1 . Os gráficos representativos do IHO para cada camada de solo, utilizando de 2 e 3
MPa como valores críticos ao desenvolvimento das culturas, são apresentados, respectivamente, nas figuras 1 e 2 .

O solo, com pouca diferenciação entre as camadas estudadas, é argiloso, apresenta porosidade total (PT) acima de $0,50 \mathrm{~m}^{3} \mathrm{~m}^{-3}$ e densidade em torno de 1,32 $\mathrm{Mg} \mathrm{m}^{-3}$, com a porosidade formada principalmente por microporos. O valor do IHO, entretanto, diminui em profundidade, principalmente quando se considerou $2 \mathrm{MPa}$ como valor crítico da RP.

Os coeficientes ajustados para os modelos de curvas de retenção de água (CRA) e de resistência do solo à penetração (CRS) foram significativos, pois o intervalo de confiança (95 \%) desses não incluiu o valor zero (Glantz \& Slinker, 1990), e apresentaram comportamento similar àqueles encontrados em outros trabalhos (Silva et al., 1994; Tormena et al., 1998b; Calonego et al., 2011; Medeiros et al., 2011). Os coeficientes de determinação indicam que a CRA e a CRS explicaram pelo menos 78 e $76 \%$, respectivamente, da variabilidade da retenção de água e da RP (Quadro 2).

Com a utilização da RPcrítica igual a $2 \mathrm{MPa}$, o limite superior do IHO foi o $\theta$ cc, nas camadas de 0,00-0,10, 0,10-0,20 e 0,20-0,30 m (Figura 1). Considerando-se a RPcrítica de $3 \mathrm{MPa}$, o $\theta_{\mathrm{CC}}$ foi o limite superior do IHO na maior faixa de variação da Ds (Figura 2), sendo substituído pela $\theta_{\mathrm{PA}}$ apenas nas Ds mais elevadas. Na camada de 0,00-0,10 m, o $\theta_{\mathrm{PA}}$ substituiu o $\theta_{\mathrm{CC}}$ quando os valores da Ds foram superiores a $1,42 \mathrm{Mg} \mathrm{m}^{-3}$. Na camada de $0,10-0,20 \mathrm{~m}$, o $\theta_{\mathrm{PA}}$ foi o fator limitante a partir da Ds de $1,47 \mathrm{Mg} \mathrm{m}^{-3}$ e na profundidade de 0,20-0,30 m, a partir da Ds de $1,45 \mathrm{Mg} \mathrm{m}^{-3}$. Quando o IHO é limitado pelo $\theta_{\mathrm{PA}}$, a aeração do solo passa a restringir o desenvolvimento da planta, geralmente por excesso de umidade. Entretanto, embora a microporosidade seja predominante, os macroporos do solo ocupam, em média, mais que $0,010 \mathrm{~m}^{3} \mathrm{~m}^{-3}$ (Quadro 1 ).

O limite inferior do IHO, considerando a RPcrítica de $2 \mathrm{MPa}$, foi o $\theta_{\mathrm{RP}}$ nas três camadas avaliadas (Figura 1), em toda a faixa de variação da Ds. Considerando-se a RPcrítica de $3 \mathrm{MPa}$ (Figura 2), o $\theta_{\mathrm{RP}}$ também foi o limite inferior do IHO nas camadas $0,10-0,20$ e 0,20-0,30 m. Somente na camada de $0,00-0,10 \mathrm{~m}$ a $\theta_{\mathrm{PMP}}$ aparece como limite inferior, para valores de Ds abaixo de $1,26 \mathrm{Mg} \mathrm{m}^{-3}$.

Nas avaliações feitas em solos cultivados, os limites superior e inferior do IHO são, em geral, a $\theta_{\mathrm{CC}}$ e a $\theta_{\mathrm{RP}}$, respectivamente, como evidenciam os trabalhos de Tormena et al. (1998b), em um Latossolo Vermelho com elevado teor de argila; Imhoff et al. (2001), em Argissolo de textura média; Silva (2003), em Argissolo arênico, Latossolo Vermelho distroférrico e Latossolo Vermelho distrófico; Beutler et al. (2004), em Latossolo Vermelho textura média e argilosa; e Klein \& Camara (2007), em um Latossolo Vermelho textura argilosa. No entanto, em uma área de mata secundária, em Latossolo Vermelho de textura argilosa, Klein \& 
Quadro 1. Valores médios dos atributos físicos do solo nas camadas de 0,00-0,10, 0,10-0,20 e 0,20-0,30 m em um Latossolo Vermelho férrico, com cultivo de grãos em sistema semeadura direta por 25 anos

\begin{tabular}{|c|c|c|c|c|c|c|c|c|}
\hline Camada & Argila & PT & Macro & Micro & Ds & Dp & $\mathrm{IHO}_{2 \mathrm{MPa}}$ & $\mathrm{IHO}_{3 \mathrm{MPa}}$ \\
\hline $\mathrm{m}$ & $\mathrm{g} \mathrm{kg}^{-1}$ & 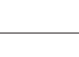 & $-\mathrm{m}^{3} \mathrm{~m}^{-3}$ & & -1 & & $-\mathrm{m}$ & \\
\hline $0,00-0,10$ & 0,578 & 0,56 & 0,21 & 0,35 & 1,30 & 2,79 & 0,083 & 0,091 \\
\hline $0,10-0,20$ & 0,577 & 0,53 & 0,18 & 0,35 & 1,34 & 2,81 & 0,049 & 0,082 \\
\hline $0,20-0,30$ & 0,578 & 0,53 & 0,17 & 0,35 & 1,32 & 2,81 & 0,034 & 0,067 \\
\hline
\end{tabular}

PT: porosidade total; Macro: macroporosidade; Micro: microporosidade; Ds: densidade do solo; Dp: densidade de partículas; e IHO: intervalo hídrico ótimo considerando 2 e $3 \mathrm{MPa}$ como valor crítico de resistência do solo à penetração.
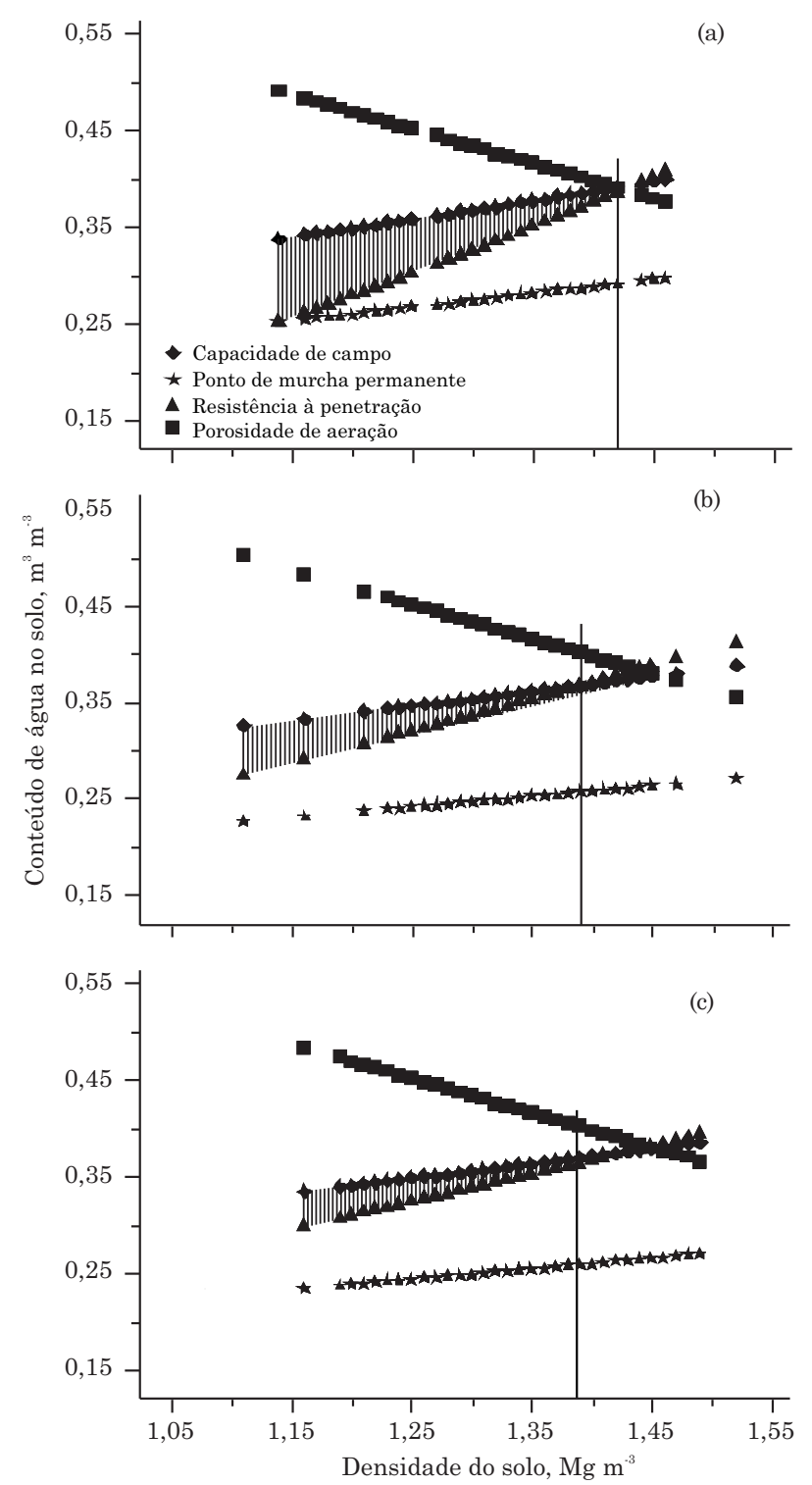

Figura 1. Variação do conteúdo volumétrico de água em um Latossolo Vermelho sob sistema semeadura direta por 25 anos em função da densidade do solo para os limites críticos de capacidade de campo, ponto de murcha permanente, porosidade de aeração de $10 \%$ e resistência do solo à penetração de 2,0 MPa, nas camadas (a) 0,00-0,10, (b) 0,10-0,20 e (c) 0,20-0,30 m. A área hachurada representa o Intervalo Hídrico Ótimo.
Libardi (2000) obtiveram como limite inferior $\theta_{\mathrm{PMP}}$. $\mathrm{O}$ fato de o $\theta_{\mathrm{RP}}$ ter sido o limite inferior do IHO também neste trabalho pode estar relacionado à compactação do solo causada pelo seu não revolvimento no SSD de longa duração, ou seja, reflete o histórico de uso do solo. E, assim, como o $\theta_{\mathrm{PMP}}$ não é o limite inferior em nenhum valor de densidade, a cultura já apresentaria restrições em seu desenvolvimento por causa da impedância mecânica imposta ao sistema radicular.

Os valores numéricos do IHO têm sido pouco utilizados para interpretar as condições físicas de campo em que foram medidas. Assim, os valores médios obtidos de 0,034 a $0,091 \mathrm{~m}^{3} \mathrm{~m}^{-3}$ (Quadro 1) evidenciam as diferenças entre camadas de solo, revelando maiores restrições hídricas nas camadas de $0,10-0,20$ e de $0,20-0,30 \mathrm{~m}$, em relação à camada de 0,00-0,10 m, mas não apresentam nenhum indicador ou limite.

A interpretação do significado IHO para o manejo dos solos tem sido mais comumente feita pelo valor de Ds em que o IHO torna-se nulo, denominado por Silva et al. (1994) como a densidade crítica do solo (Dsc). Neste trabalho, utilizando-se o valor de RPcrítica de $2 \mathrm{MPa}$, a Dsc foi de $1,42 \mathrm{Mg} \mathrm{m}^{-3}$ para a camada de 0,00-0,10 m. Nas camadas de 0,10-0,20 e 0,20-0,30 m, os valores de Dsc foram, respectivamente 1,39 e $1,41 \mathrm{Mg} \mathrm{m}^{-3}$. Considerando-se a RPcrítica igual a $3 \mathrm{MPa}$, foram obtidos valores de Dsc de $1,48 \mathrm{Mg} \mathrm{m}^{-3}$ para a camada de $0,00-0,10 \mathrm{~m}$ e de $1,51 \mathrm{Mg} \mathrm{m}^{-3}$, para as camadas de 0,10-0,20 e 0,20-0,30 m.

Os valores da Dsc variam com o tipo de solo. Silva (2003) verificou que a Dsc foi de $1,80 \mathrm{Mg} \mathrm{m}^{-3}$ para um Argissolo Vermelho-Amarelo de textura arenosa e $1,40 \mathrm{Mg} \mathrm{m}^{-3}$, para um Latossolo Vermelho distroférrico muito argiloso. Reichert et al. (2009), compilando dados da literatura, apontaram que o valor da Dsc tem relação inversa com o teor de argila do solo, estando relacionado portanto ao tipo de solo. Esses autores afirmaram que a Dsc é também influenciada pelo manejo do solo, como observado por Pereira et al. (2010), que obtiveram valores de Dsc de $1,43 \mathrm{Mg} \mathrm{m}^{-3}$ para cultivo convencional e valores mais altos e mais baixos que esse para diferentes sistemas de semeadura direta, em um Latossolo Vermelho. Calonego et al. (2011) obtiveram aumento da Dsc com utilização de plantas com sistema radicular mais vigoroso. 
As relações do IHO com a Ds, considerando os valores de RPcrítica de 2 e $3 \mathrm{MPa}$, são apresentadas respectivamente nas figuras 3 e 4 . Verifica-se diminuição do IHO em razão do aumento da Ds, em
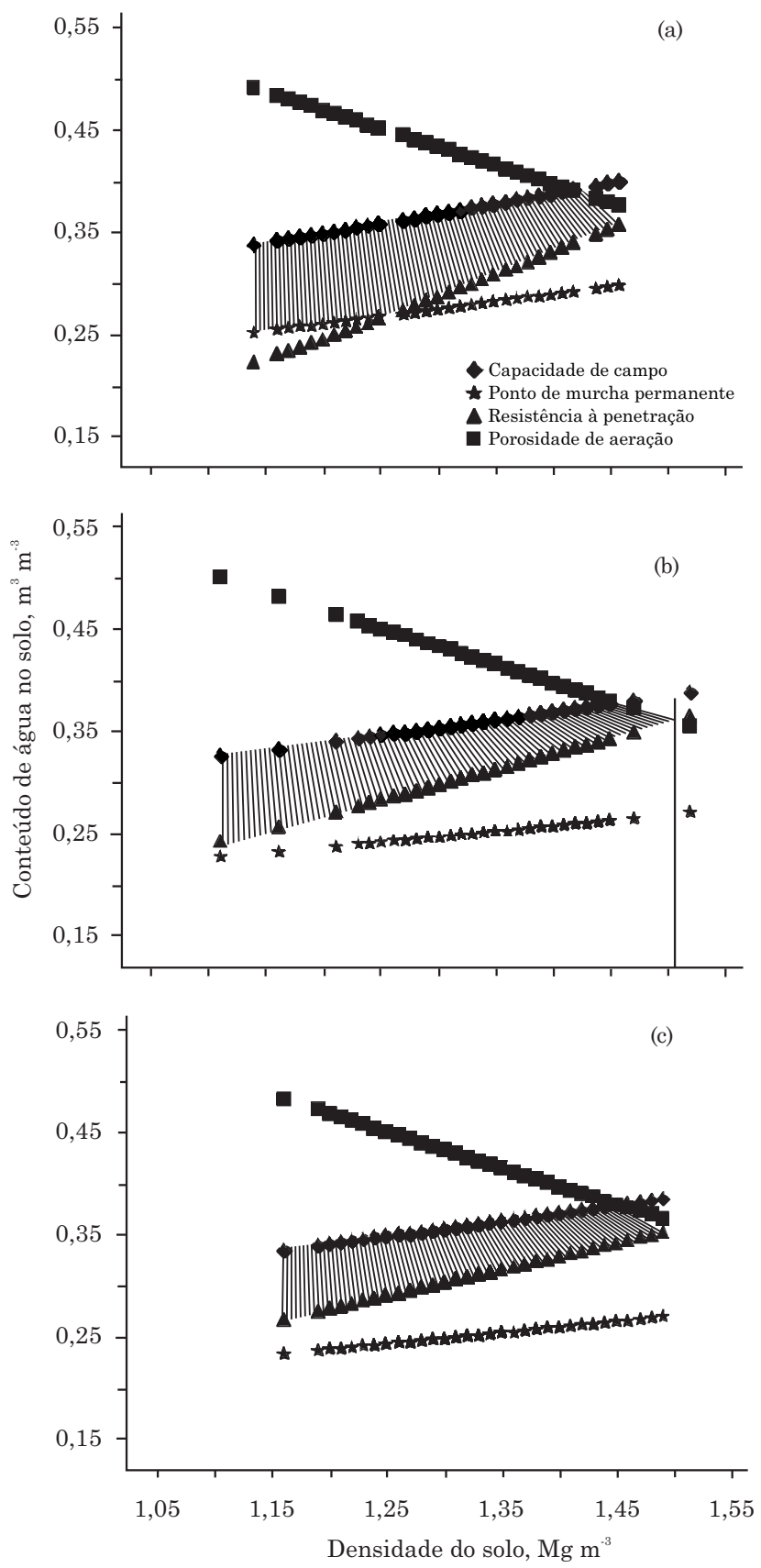

Figura 2. Variação do conteúdo volumétrico de água em um Latossolo Vermelho sob sistema semeadura direta por 25 anos em função da densidade do solo para os limites críticos de capacidade de campo, ponto de murcha permanente, porosidade de aeração de $10 \% \mathrm{e}$ resistência do solo à penetração de $3,0 \mathrm{MPa}$, nas camadas (a) 0,00-0,10, (b) 0,10-0,20 e (c) $0,20-0,30$ m. A área hachurada representa o Intervalo Hídrico Ótimo. todas as três camadas de solo avaliadas, efeito encontrado também por Silva (2003) e Tormena et al. (2007). Nota-se que com a RPcrítica de $2 \mathrm{MPa}$ essa relação é representada por um único segmento e reta (Figura 3). Já com a RPcrítica de $3 \mathrm{MPa}$, a relação entre Ds e IHO modifica-se nas densidades em que o $\theta_{\mathrm{PA}} \mathrm{e}, \mathrm{ou}, \mathrm{o} \theta_{\mathrm{RP}}$ passam a determinar a disponibilidade de água no solo (Figura 4). Na camada 0,00-0,10 m, o IHO aumenta até a Ds em que o $\theta_{\mathrm{RP}}$ substitui o $\theta_{\mathrm{PMP}}$. Assim, com a utilização da RPcrítica de $3 \mathrm{MPa}$, foram identificados valores da Ds em que $\theta_{\mathrm{RP}}$ e $\theta_{\mathrm{PA}}$ tornamse os limites do IHO, indicando os fatores que restringem a disponibilidade de água no solo em condição de maior grau de compactação.

A escolha do valor da RPcrítica tem sido discutida por outros autores, com implicações para a determinação da Dsc. Klein \& Camara (2007) utilizaram RPcrítica iguais a 2 e 3 MPa e concluíram que, para o cultivo da soja em SSD, o valor de $3 \mathrm{MPa}$ foi mais adequado para refletir o crescimento da cultura. Kaiser et al. (2009), estudando um Latossolo Vermelho argiloso, encontraram valores de Dsc de 1,36 a $1,49 \mathrm{Mg} \mathrm{m}^{-3}$, quando utilizados valores de RPcrítica entre 1,5 e 3,0 $\mathrm{MPa}$, e verificaram que as raízes de feijoeiro não cresceram quando o IHO foi nulo, utilizando RPcrítica de $3 \mathrm{MPa}$. Reichert et al. (2009) afirmaram que a RPcrítica de $2 \mathrm{MPa}$ não é apropriada para solo com manejo SSD, uma vez que as plantas crescem ainda que o IHO seja nulo.

Diversos autores apresentam valores críticos de densidade do solo para a produtividade ou para o crescimento do sistema radicular de diversas culturas. Beutler et al. (2004), por exemplo, observaram que a produtividade da soja e do arroz foi reduzida a partir da Ds de $1,27 \mathrm{Mg} \mathrm{m}^{-3}$, valor abaixo da Dsc determinada pelo IHO, e Reichardt \& Timm (2004) afirmaram que os limites de Ds para um bom crescimento vegetal variam de 1,0 a $1,4 \mathrm{Mg} \mathrm{m}^{-3}$.

No campo experimental podem existir, então, áreas com limitações ao crescimento das culturas, principalmente na camada de 0,10-0,20 m, com valores médios de $\mathrm{Ds}$ de $1,34 \mathrm{Mg} \mathrm{m}^{-3}$. No quadro 3 , são apresentados, respectivamente, o resumo estatístico dos dados de Ds nos 102 pontos da área experimental e os parâmetros de ajuste dos semivariogramas obtidos em cada camada de solo. Nas três camadas, os valores variam entre $1,14 \mathrm{e} 1,51 \mathrm{Mg} \mathrm{m}^{-3}$, com baixo coeficiente de variação $(\mathrm{CV}<12 \%)$. O resultado da análise da semivariância indicou dependência espacial, com razão de dependência espacial alta para a camada de 0-0,10 m e média para as camadas de 0,10-0,20 e de 0,20-0,30 m. Os valores do alcance e da variância estruturada aumentaram com a profundidade, indicando maior homogeneidade da Ds na superfície.

Os mapas de variabilidade espacial da Ds para as camadas de 0,00-0,10, 0,10-0,20 e 0,20-0,30 m são apresentados na figura 5 . Os valores da Ds tendem a aumentar em direção à posição inferior do campo 
experimental, e a proporção da área com valores mais elevados é maior nas camadas 0,10-0,20 e 0,20-0,30 m.

Utilizando-se o valor de RPcrítica de $3 \mathrm{MPa}$ (Dsc de $1,48 \mathrm{Mg} \mathrm{m}^{-3}$ para a camada mais superficial e Dsc de $1,51 \mathrm{Mg} \mathrm{m}^{-3}$ para as outras camadas de solo), nenhum ponto da área experimental apresentou Ds $>$ Dsc. Na figura 6, são apresentados os mapas de variabilidade espacial em que a Ds foi menor ou maior que a Dsc nas camadas de 0,00-0,10 e 0,10-0,20 m, considerando-se o valor de RPcrítica de $2 \mathrm{MPa}$. Os mapas não são coincidentes, indicando ser importante

Quadro 2. Modelos de regressão não-linear das curvas de retenção de água (CRA) e de resistência do solo à penetração (CRS) em um Latossolo Vermelho férrico, com cultivo de grãos em sistema semeadura direta por 25 anos

\begin{tabular}{ccc}
\hline Camada & Curva de retenção de água no solo & Curva de resistência do solo à penetração \\
\hline $\mathrm{m}$ & & \\
$0,00-0,10$ & $\theta=\exp (-1,4054+0,5202 \mathrm{Ds}) \psi^{-0,0582} ; \mathrm{R}^{2}=0,78^{* * *}$ & $\mathrm{RP}=0,0147 \theta^{-3,0453} \mathrm{Ds}^{5,8338} ; \mathrm{R}^{2}=0,76^{* * *}$ \\
$0,10-0,20$ & $\theta=\exp (-1,2656+0,4291 \mathrm{Ds}) \psi^{-0,0710} ; \mathrm{R}^{2}=0,85^{* * *}$ & $\mathrm{RP}=0,0203 \theta^{-3,2519} \mathrm{Ds}^{4,1517} ; \mathrm{R}^{2}=0,75^{* * *}$ \\
$0,20-0,30$ & $\theta=\exp (-1,2583+0,4257 \mathrm{Ds}) \psi^{-0,0707} ; \mathrm{R}^{2}=0,81^{* * *}$ & $\mathrm{RP}=0,0154 \theta^{-3,5780} \mathrm{Ds}^{3,9057} ; \mathrm{R}^{2}=0,76^{* * *}$ \\
\hline
\end{tabular}

RP: resistência do solo à penetração $(\mathrm{MPa})$; Ds: densidade do solo $\left(\mathrm{Mg} \mathrm{m}^{-3}\right) ; \theta$ : umidade volumétrica do solo $\left(\mathrm{m}^{3} \mathrm{~m}^{-3}\right) ; \psi$ : potencial mátrico $(|\mathrm{hPa}|) ; \mathrm{R}^{2}$ : coeficiente de determinação. ${ }^{* * *}$ : significativo a $1 \%$ pelo teste $\mathrm{F}$.

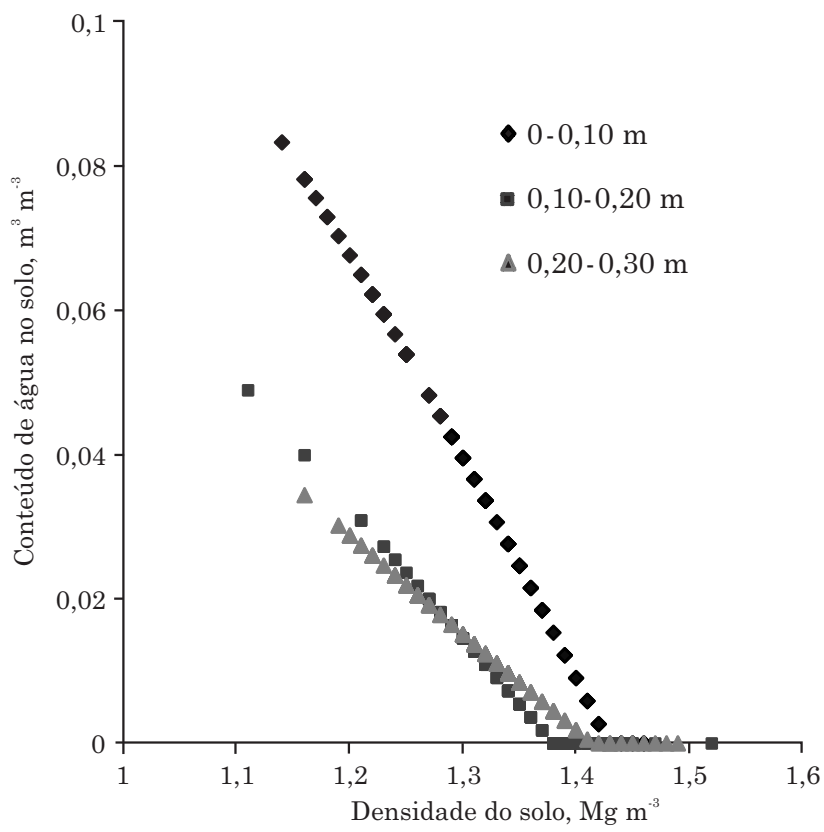

Figura 3. Variação do Intervalo Hídrico Ótimo em função da densidade do solo, para a resistência à penetração de 2,0 MPa, nas camadas 0,00-0,10, 0,10-0,20 e 0,20-0,30 m em um Latossolo Vermelho sob sistema semeadura direta por 25 anos.

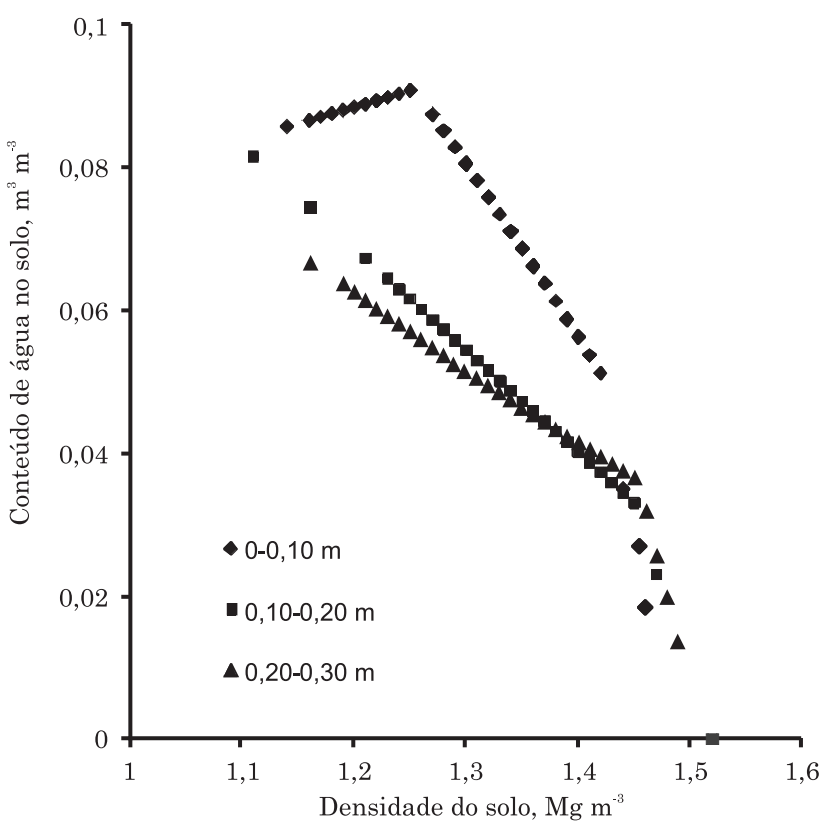

Figura 4. Variação do Intervalo Hídrico Ótimo em função da densidade do solo para a resistência à penetração de $3,0 \mathrm{MPa}$, nas camadas $0,00-0,10$, 0,10-0,20 e 0,20-0,30 m em um Latossolo Vermelho sob sistema semeadura direta por 25 anos.

Quadro 3. Parâmetros estatísticos da variabilidade e ajustes do semivariograma da densidade do solo (Ds, $\mathrm{Mg} \mathrm{m}^{-3}$ ) nas três camadas de um Latossolo Vermelho sob sistema semeadura direta por 25 anos

\begin{tabular}{|c|c|c|c|c|c|c|c|c|c|c|c|c|}
\hline Variável & Média & DP & CV & Mín. & Máx. & Assim. & Curtose & Modelo & $\mathrm{C}_{0}$ & $\mathrm{C}_{1}$ & $\mathbf{a}$ & RD \\
\hline & & & $\%$ & & & & & & & & $\mathrm{~m}$ & \\
\hline $\mathrm{Ds}_{0,0-0,10 \mathrm{~m}}$ & 1,30 & 0,077 & 5,90 & 1,14 & 1,46 & 0,293 & $-0,040$ & Exponencial & 0,0051 & 0,0395 & 36,24 & Alta \\
\hline $\mathrm{Ds}_{0,10-0,20 \mathrm{~m}}$ & 1,34 & 0,064 & 4,83 & 1,16 & 1,52 & $-0,386$ & 0,825 & Exponencial & 0,0370 & 0,0592 & 74,57 & Média \\
\hline $\mathrm{Ds}_{0,20-0,30 \mathrm{~m}}$ & 1,32 & 0,067 & 5,07 & 1,16 & 1,49 & 0,044 & 0,283 & Esférico & 0,0686 & 0,0684 & 86,28 & Média \\
\hline
\end{tabular}

DP: desvio-padrão; CV: coeficiente de variação; Mín: valor mínimo; Máx: valor máximo; Assim.: assimetria; $\mathrm{C}_{0}$ : efeito pepita; $\mathrm{C}_{1}$ : variância estruturada; a: alcance; e RD: razão de dependência espacial. 
(a)

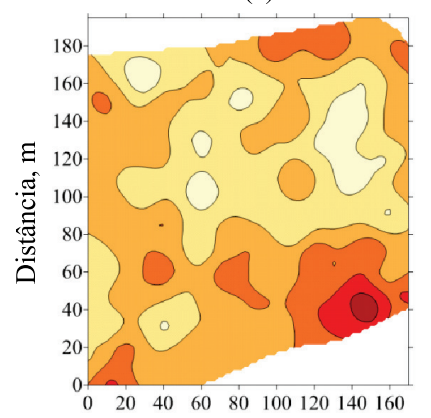

(b)

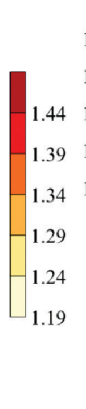

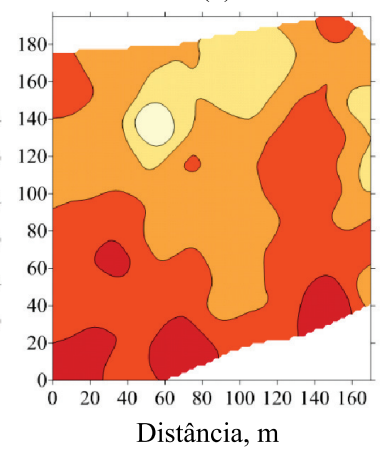

(c)

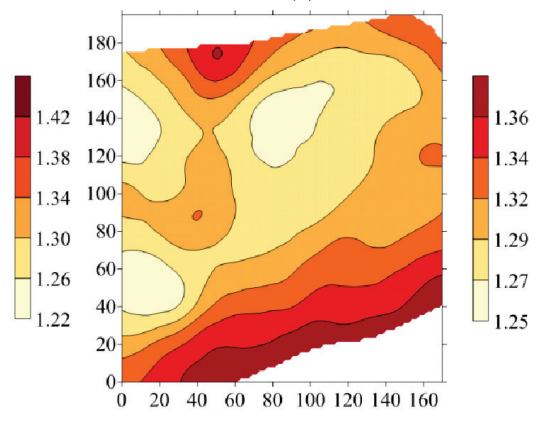

Figura 5. Mapas de variabilidade espacial da densidade do solo $\left(\mathrm{Mg} \mathrm{m}^{-3}\right)$ em um Latossolo Vermelho sob sistema semeadura direta por 25 anos, nas camadas de 0,00-0,10 (a), 0,10-0,20 (b) e 0,20-0,30 m (c).

(a)

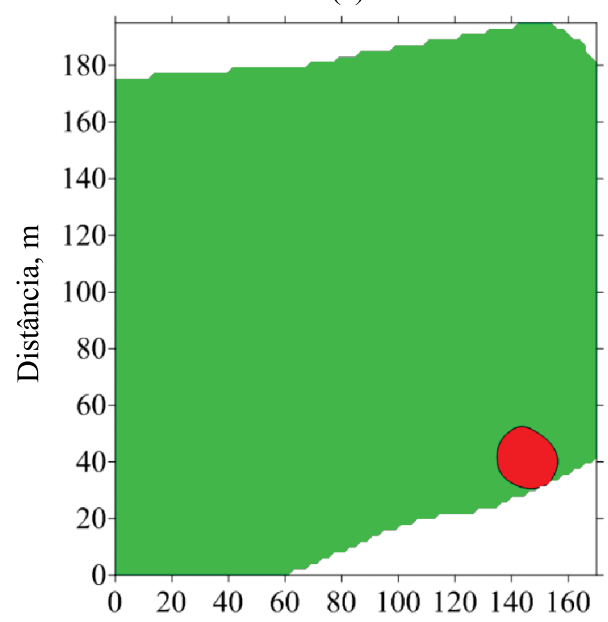

(b)

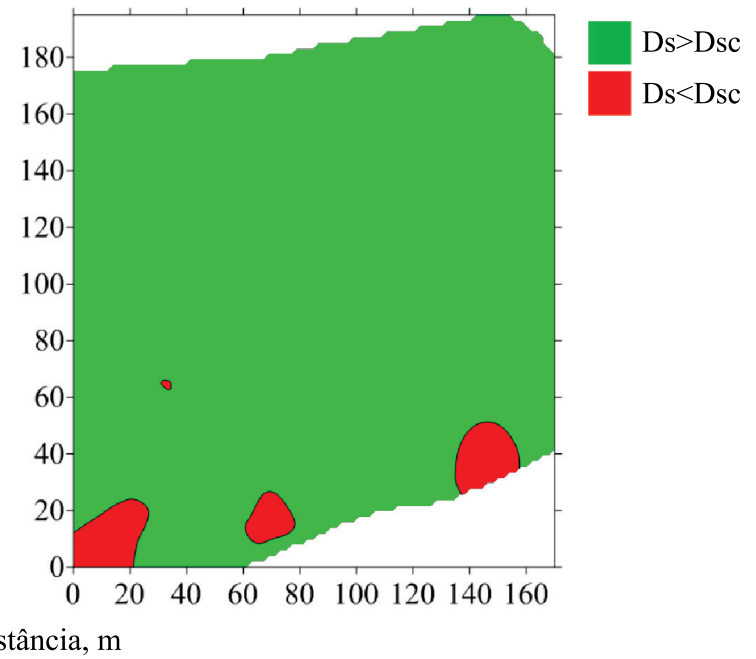

Figura 6. Mapas da distribuição espacial da relação entre Densidade crítica do solo (Dsc) e densidade do solo (Ds) em um Latossolo Vermelho sob sistema semeadura direta por 25 anos, nas camadas de 0,000,10 (a) e 0,10-0,20 m (b), considerando-se 2 MPa como valor crítico de resistência do solo à penetração.

realizar essa avaliação em diferentes camadas ou, pelo menos, nas onde há maior efeito do manejo na alteração da estrutura do solo, muitas vezes na entre $0,10-0,20 \mathrm{~m}$.

Com esses mapas, podem-se visualizar os locais menos favoráveis ao crescimento das culturas, considerando-se a Ds > Dsc, localizadas na porção inferior e à direita do campo experimental. Entre as razões para o aparecimento dessas áreas menos favoráveis, pode estar um maior grau de compactação causado principalmente por tráfego ou manobras mais frequentes de máquinas associado a variações inerentes ao solo, uma vez que a porção inferior da vertente está sujeita a maiores conteúdos de água no solo.

Nesses locais menos favoráveis ao crescimento das culturas, onde o IHO tende a ser nulo, devem-se propor ações corretivas ou mudança no sistema de manejo para recuperação da qualidade do solo. Com a utilização do valor RPcrítica de $2 \mathrm{MPa}$, foi possível identificar no mapa de variabilidade espacial da área experimental pontos prioritários para intervenção ou alteração do manejo do solo.

\section{CONCLUSÕES}

1. A área de estudo evidencia redução da disponibilidade de água, uma vez que $\theta_{\mathrm{RP}}$ foi o limite inferior do IHO em quase toda a faixa de variação da Ds, nas três camadas de solo estudadas.

2. A escolha do valor crítico da resistência do solo à penetração interfere nos valores de IHO e Dsc, mas pode auxiliar na interpretação dos resultados experimentais.

3. O IHO associado a mapas de variabilidade espacial da Ds, para determinação de pontos em que a Ds é maior que a Dsc, é ferramenta para auxiliar a tomada de decisão para intervenção ou modificação do manejo do solo. 
4. O uso do valor de RPcrítica de $3 \mathrm{MPa}$ possibilitou identificar valores da Ds em que $\theta_{\mathrm{RP}}$ e $\theta_{\mathrm{PA}}$ tornam-se os limites inferior e o superior, respectivamente, do IHO, indicando restrição à disponibilidade de água no solo em condição de maior grau de compactação.

5. O uso do valor de RPcrítica de 2 MPa possibilitou identificar no mapa de variabilidade espacial da Ds da área experimental os pontos prioritários para intervenção ou alteração do manejo.

\section{LITERATURA CITADA}

BERTOL, I.; ALBUQUERQUE, J.A.; LEITE, D.; AMARAL, A.J. \& ZOLDAN JUNIOR, W.A. Propriedades físicas do solo sob preparo convencional e semeadura direta em rotação e sucessão de culturas, comparadas às do campo nativo. R. Bras. Ci. Solo, 28:155-163, 2004.

BEUTLER, A.N.; CENTURION, J.F. \& SILVA, A.P. Intervalo hídrico ótimo e a produção da soja e arroz em dois Latossolos. Irriga, 9:181-192, 2004.

BUSSCHER, W.J. Adjustment of flat-tipped penetrometer resistance data to a common water content. Am. Soc. Agron. Eng., 33:519-524, 1990.

CALONEGO, J.C.; BORGHI, E. \& CRUSCIOL, C.A.C. Intervalo hídrico ótimo do solo com cultivo consorciado de milho e braquiária. R. Bras. Ci. Solo, 35:2183-2190, 2011.

CAVALIERI, K.M.V.; TORMENA, C.A.; VIDIGAL FILHO, P.S.; GONÇALVES, A.C.A. \& COSTA, A.C.S. Efeitos de sistemas de preparo nas propriedades físicas de um Latossolo Vermelho distrófico. R. Bras. Ci. Solo, 30:137$147,2006$.

COSTA, F.S.; ALBUQUERQUE, J.A.; BAYER, C.; FONTOURA, S.M.V. \& WOBETO, C. Propriedades físicas de um Latossolo Bruno afetadas pelos sistemas plantio direto e convencional. R. Bras. Ci. Solo, 27: 527-535, 2003.

DE MARIA, I.C.; CASTRO, O.M. \& DIAS, H.S. Atributos físicos do solo e crescimento radicular de soja em Latossolo Roxo sob diferentes métodos de preparo do solo. R. Bras. Ci. Solo, 23:703-709, 1999.

DORAN, J.W. \& PARKIN, T.B. Defining and assessing soil quality. In: DORAN, J.W.; COEMAN, D.C.; BEZDICEK, D.F. \& STEWART, B.A., eds. Defining soil quality for sustainable environment. Madison, Soil Science Society of America, 1994. p.3-21. (SSSA Special Publication, 35)

FREDDI, O.S.; CENTURION, J.F.; ARATANI, R.G. \& BEUTLER, A.N. Compactação do solo e intervalo hídrico ótimo no crescimento da parte aérea e produtividade da cultura do milho. Irriga, 13:272-287, 2008.

IMHOFF, S.; SILVA, A.P.; DIAS JUNIOR, M.S. \& TORMENA, C.A. Quantificação de pressões críticas para o crescimento das plantas. R. Bras. Ci. Solo, 25:11-18, 2001.

GLANTZ, S.A. \& SLINKER, B.K. Primer of applied regression and analysis of variance. New York, McGraw-Hill, 1990. $777 \mathrm{p}$.
KAISER, D.R.; REINERT, D.J.; REICHERT, J.M.; COLLARES, G.L. \& KUNZ, M. Intervalo hídrico ótimo no perfil explorado pelas raízes de feijoeiro em um Latossolo sob diferentes níveis de compactação. R. Bras. Ci. Solo, 33:845$855,2009$.

KLEIN, V.A. \& CAMARA, R.K. Rendimento da soja e intervalo hídrico ótimo em Latossolo Vermelho sob plantio direto escarificado. R. Bras. Ci. Solo, 31:221-227, 2007.

KLEIN, V.A. \& LIBARDI, P.L. Faixa de umidade menos limitante ao crescimento vegetal e sua relação com a densidade do solo ao longo do perfil de um Latossolo Roxo. Ci. Rural, 30:959-964, 2000.

LEÃO, T.P.; SILVA, A.P.; MACEDO, M.C.M.; IMHOFF, S. \& EUCLIDES, V.P.B. Intervalo hídrico ótimo na avaliação de sistemas de pastejo contínuo e rotacionado. R. Bras. Ci. Solo, 28:415-423, 2004.

MEDEIROS, J.C.; SILVA, A.P.; CERRI, C.E.P.; GIAROLA, N.F.B.; FIGUEIREDO, G.C. \& FRACETTO, F.J.C. Linking physical quality and $\mathrm{CO}_{2}$ emissions under longterm no-till and conventional-till in a subtropical soil in Brazil. Plant Soil, 338:5-15, 2011.

PEREIRA, F.S.; ITAMAR ANDRIOLI, I.; BEUTLER, A.N.; ALMEIDA, C.X. \& PEREIRA, F.S. Physical quality of an Oxisol cultivated with maize submitted to cover crops in the precropping period. R. Bras. Ci. Solo, 34:211-218, 2010.

REICHARDT, K. \& TIMM, L.C. Solo, planta e atmosfera: Conceitos, processos e aplicações. São Paulo, Manole, 2004. $478 \mathrm{p}$.

REICHERT, J.M.; SUZUKI, L.E.A.S.; REINERT, D.J.; HORN, R. \& HÅKANSSON, I. Reference bulk density and critical degree-of-compactness for no-till crop production in subtropical highly weathered soils. Soil Till. Res., 102:242$254,2009$.

SETZER, J. Atlas climático e ecológico do Estado de São Paulo. Comissão Interestadual da Bacia Paraná-Uruguai, 1966. 61p.

SANTOS, H.G.; JACOMINE, P.K.T.; ANJOS, L.H.C.; OLIVEIRA, V.A.; OLIVEIRA, J.B.; COELHO, M.R.; LUMBRERAS, J.F. \& CUNHA, T.J.F., eds. Sistema brasileiro de classificação de solos. 2.ed. Rio de Janeiro, Embrapa Solos, 2006. 306p.

SILVA, A.P.; KAY, B.D. \& PERFECT, E. Characterization of the least limiting water range. Soil Sci. Soc. Am. J., 58:1775-1781, 1994.

SILVA, V.R. Propriedades físicas e hídricas em solos sob diferentes estados de compactação. Santa Maria, Universidade Federal de Santa Maria, 2003. 171p. (Tese de Doutorado). Disponível em: <http:// cascavel.cpd.ufsm.br/tede/tde_busca/index.php>.

TORMENA, C.A.; ARAÚJO, M.A. \& FIDALSKI, J. Variação temporal do intervalo hídrico ótimo de um Latossolo Vermelho distroférrico sob sistema de plantio direto. R. Bras. Ci. Solo, 31:211-219, 2007.

TORMENA C.A.; ROLOFF, G. \& SÁ, J.C.M. Propriedades físicas do solo sob plantio direto influenciadas por calagem, preparo inicial e tráfego. R. Bras. Ci. Solo, 22:301-309, 1998a. 
TORMENA, C.A.; SILVA, A.P. \& LIBARDI, P.L. Caracterização do intervalo hídrico ótimo de um Latossolo Roxo sob plantio direto. R. Bras. Ci. Solo, 22:573-581, 1998 b.
VIEIRA, S.R. Geoestatística em estudos de variabilidade espacial do solo. In: NOVAIS, R.F.; ALVAREZ V., V.H. \& SHAEFER, C.E.G.R., eds. Tópicos em ciência do solo. Viçosa, MG, Sociedade Brasileira de Ciência do solo, 2000. v.1, p.1-54. 